\section{Bold perspective}

Ion-selective Electrodes. (Cambridge Monographs in Physical Chemistry Volume 2.) By Jiri Koryta. Pp. 207. (Cambridge University : Cambridge and London, August 1975.) $£ 10.50$.

THE development and application of ion-selective electrodes (ISEs) based on membrane electrochemical principles have been, with comparable activity in liquid chromatography, the two most rapidly advancing areas in chemical analysis and continuous monitoring. Since 1965 improved or new measurements have become possible which have not only given impetus to analytical potentiometry, but have a broad impact in diverse areas as environmental science, physiology, clinical chemistry and biophysics. In fact one difficulty in following progress in the field is the widespread use of ISEs

\section{Nuclear structure}

Structure of the Nucleus. By M. A. Preston and R. K. Bhaduri. Pp. xivt 693. (Addison-Wesley: Reading, Massachusetts and London, August 1975.) Cloth \$29.50; Paper \$19.50.

FOR more than a decade Preston's Physics of the Nucleus has been used by nuclear physicists as a comprehensive and reliable reference book. Its essential theme is the attempt to derive the properties of the nucleus from our knowledge of the nucleonnucleon forces. This can be done to some extent, but in many respects we still have to rely on phenomenological models. The years since 1962 have seen many important advances, and while progress has been made in some areas the problem is now seen to be more complicated than was previously thought, particularly because of the importance of many-body forces. To deal with this situation Professor Preston, now joined by Dr. Bhaduri, has thoroughly revised and re-written the chapters dealing with nuclear structure, and since this is already as long as the whole book before revision the chapters on nuclear reactions have been omitted. The plan is very similar and successive chapters deal with the constituents of nuclei, internucleon forces, nuclear moments, nuclear shapes and sizes, and nuclear binding energies. The second part is devoted to nuclear models, correlation in nuclear matter, collective nuclear motion, and Hartree-Fock and particle-hole calculations. The final part covers $\alpha$ radioactivity and fission. The standard of the original volume is amply maintained, and the new version will be a valuable and much-used text for years to come. P. E. Hodgson and the large number of specialist journals in which results appear. ISEs are serving the fundamental purposes of making analytical chemistry visible in other branches of chemistry, physics, medical and health sciences and the converse effect of broadening the interests of analytical chemists in interdisciplinary problems and projects.

To write a book at midstream in a rapidly moving field requires courage, and to achieve a sense of perspective on the past and possible future developments is most unusual. Koryta's new volume is well organised and executed, and contains clearly presented fundamentals and applications drawn from all fields touched by ISEs. Jiri Koryta is a well known electrochemist, a major disciple of Nobel Laureate J. Heyrovsky and an exponent of the polarographic method. His outstanding contributions to theory and practice of coupled homogeneoushetergeneous electrode processes, complex ion equilibria and adsorption effects make him an ideal commentator on the field of ISEs and membrane processes. The primary strength of the book is the mature overview given on the topics of diffusion-migration and the interfacial processes which underlie the potential sources for fixed site, mobile site and neutral carrier classes of membrane electrodes. At the same time practical, analytical methods are tabulated and current topics such as continuous monitoring and use of computercontrolled titrations are described.

Only on hindsight can one determine key, definitive volumes on a given speciality area. Because of the continuing growth in this field, all previous books, although useful for reference, are clearly premature. This new book unfortunately must suffer from the same limitations: new fundamental studies on response mechanisms, time-dependence of responses and applications are still appearing in current literature at a rapid rate. This volume contains literature citations only as recent as 1973 and is an expansion of Koryta's excellent 1972 review article. Nevertheless, this book is the finest, comprehensive work published to date.

Richard P. Buck

\section{Climatic change}

Ice Ages: Ancient and Modern. (Proceedings of the 21st Inter-University Congress, January 1974.) Edited by A. E. Wright and F. Mosley. Pp. 320. (Seel House : Liverpool, 1975.) £12.00; $\$ 33.00$.

THIS book is advertised as suitable for geology and geography students "from sixth form to final year undergraduate level"; but unless the sixth formers of today are markedly more able than those of my generation it would seem better suited to students of Earth sciences at a rather later stage of their academic careers, including those active in research. For such people, the book will be of most value to those who have had a less formal education in geography and geology at an earlier stage. It provides a good quick guide to geology and timescales, to processes of sedimentation and to glacitectonic structures, before moving on to discuss climate and climatic change in more detail.

There is one exceptionable statement early in the first section ("Introduction to the Quaternary") in which F. W. Shotton refers to ice ages occurring when ". . snowlines extend outwards from the poles, ice sheets expand, mountain glaciers push forward.

and makes no reference to the recent work which suggests strongly that ice ages can occur much more quickly than this older picture suggests, with snow and ice cover building up over a wide area of the northern hemisphere in a few years or decades. Otherwise, the various contributions (which are based on lectures given at Birmingham University in January 1974) provide a good overview of how geological and other evidence (pollen analysis, studies of fossil beetles and so on) provides an indication of how climate has fluctuated

But the discussion of the causes of such changes is less satisfactory, and the volume also suffers from being almost entirely concerned with events in north-western Europe, particularly Britain. It is inexcusable, in a book of this length, to dismiss variations caused by changes in the Earth's orbit and inclination in less than a page and by volcanic dust in three lines. There is a preponderance of geological jargon in some of the contributions, and most of the contributors share the geologists' preference for talking in terms of the named geological periods of time, rather than in years b.p. These deficiencies are balanced to some extent by the copious references and by a summary from the editors putting the various contributions into perspective. In this case, the summary is particularly valuable and does not become bogged down by attempts at verbatim reporting of question and answer sessions. The importance of this kind of research, and the slightly parochial inclination of this book, are highlighted by one titbit from the summary: "even today it would only require an average temperature of $2{ }^{\circ} \mathrm{C}$ to [produce] glaciers on Ben Nevis".

Those searching for more answers in the study of climatic changes will by stimulated by this volume.

John Gribbin 